\section{Tracer Kinetic Model of Blood-Brain Barrier Transport of Plasma Protein-bound Ligands Empiric Testing of the Free Hormone Hypothesis}

William M. Pardridge and Elliot M. Landaw Departments of Medicine, Biomathematics, and Pediatrics, University of California, Los Angeles, School of Medicine, Los Angeles, California 90024

bstract. Previous studies have shown that the fraction of hormone or drug that is plasma protein bound is readily available for transport through the brain endothelial wall, i.e., the blood-brain barrier (BBB). To test whether these observations are reconcilable with the free-hormone hypothesis, a tracer-kinetic model is used in the present investigations to analyze in vivo initial extraction data on BBB transport of proteinbound steroid hormones (dihydrotestosterone, testosterone, estradiol, and corticosterone), thyroid hormones (triiodothyronine), and lipophilic amine drugs (propranolol). The plasma proteins used are bovine albumin and human orosomucoid. Transport data was fit to a modification of the Kety-Renkin-Crone equation of capillary physiology; the modified equation incorporates the principles of both capillary physiology and plasma proteinligand mass action binding relationships. In most cases, the experimental data is best fit to the model equation when the apparent in vivo dissociation constant, $K_{\mathrm{D}}{ }^{\mathrm{a}}$, of the ligand protein binding reaction increases to values that are 5- to 50-fold greater than the in vitro dissociation constant, $K_{\mathrm{D}}$. This result indicates that the rate of ligand dissociation from the plasma protein is accelerated in the capillary bed relative to the in vitro situation. It is hypothesized that the major factor leading to the rapid transport in vivo of protein-bound ligands into tissues such as brain is an endothelial-induced decrease in the affinity of the plasma protein for the ligand. Under these

Dr. Pardridge is the recipient of a National Institutes of Health Research Career Development Award (AM-00783).

These studies were presented at the 65th Annual Meeting of the Endocrine Society, San Antonio, Texas, June 1983.

Received for publication 22 June 1983 and in revised form 30 March 1984.

J. Clin. Invest.

(c) The American Society for Clinical Investigation, Inc.

0021-9738/84/09/0745/08 \$1.00

Volume 74, September 1984, 745-752 conditions, the amount of plasma ligand available for tissue clearance in vivo parallels the protein-bound fraction, not the free hormone.

\section{Introduction}

The free-hormone hypothesis assumes that the concentration of circulating hormone that is available for uptake by tissues in vivo is equal to the concentration of free hormone measured in vitro, e.g., by equilibrium dialysis $(1,2)$. Similar precepts hold for the free-drug hypothesis (3). Implicit in these formulations is the assumption that the binding constants that govern the ligand-plasma protein interactions in vivo in the microenvironment of the tissue capillary are approximated by the binding constants observed in vitro. Previous studies from our laboratory since 1979 have reported the effects of plasma proteins on the blood-brain barrier (BBB) ${ }^{\prime}$ transport of steroid hormones (4), thyroid hormones $(5,6)$, free fatty acids (7), and drugs (8). These studies have shown that the apparent dissociation constant, $K_{\mathrm{D}}^{\mathrm{a}}$, of plasma protein-binding of ligands in vivo in the brain capillary is in most cases many times greater than the in vitro dissociation constant, $K_{\mathrm{D}}$. That is, the dissociation of ligands from plasma proteins is enhanced in the micro-environment of the living capillary such that the concentration of available (transportable) hormone in vivo is many times greater than the concentration of free hormone in vitro. On the basis of these observations we have suggested that the precepts of the free-hormone hypothesis are false (9). However, Robbins and Johnson (10) and Ekins et al. (11) have recently attempted to reconcile the free hormone hypothesis with our experimental observations. Such a reconciliation would probably require unphysiologically high membrane permeability constants, since we have observed that tissues rapidly take up hormone from the circulation in excess of the freehormone pool in plasma. The conflicting interpretations of the relevance of our data to the free-hormone hypothesis may be attributed to the lack of a mathematical model that allows

1. Abbreviations used in this paper: $A_{\mathrm{F}}$, plasma protein concentration; $\mathrm{BBB}$, blood-brain barrier; $E$, unidirectional ligand extraction; $K_{\mathrm{D}}$, dissociation constant in vitro; $K_{\mathrm{D}}$, apparent dissociation constant in vivo; $t$, mean brain capillary transit time; $\mathrm{T}_{3}$, triiodothyronine. 
for the quantitative analysis of the transport of free and bound hormones in vivo. Therefore, the purpose of the present studies was to elaborate a tracer-kinetic model and to fit the experimental data to the model given the assumptions of the opposing hypotheses. In the first case (9) (e.g., case I) it is assumed that the rate of ligand dissociation from plasma protein in vivo may be enhanced relative to the in vitro situation, and in the second case $(10,11)$ (e.g., case II) it is assumed that the dissociation rate in vivo is fixed at the in vitro value.

\section{Methods}

Carotid artery injection technique. The measurement of $E$, the unidirectional extraction, for the six ligands under study, (estradiol, testosterone, dihydrotestosterone, corticosterone, triiodothyronine $\left[\mathrm{T}_{3}\right]$, and propranolol) has been reported previously $(4,5,8)$ for barbiturateanesthetized adult rats $(200-300 \mathrm{~g})$. The ${ }^{3} \mathrm{H}$-test compound and $\left[{ }^{14} \mathrm{C}\right]$ butanol, a freely diffusible internal reference, were rapidly injected $(<1 \mathrm{~s})$ into the common carotid artery in a 150-200 $\mu$ l solution of Hepes-buffered Ringer's solution (pH 7.4) containing various concentrations of bovine albumin $(0.37-1.5 \mathrm{mM})$ or human orosomucoid $(7.5-75 \mu \mathrm{M})$. In the case of $\mathrm{T}_{3}$, the test compond was ${ }^{125} \mathrm{I}$ labeled and the reference compound was $\left[{ }^{3} \mathrm{H}\right]$ water. $15 \mathrm{~s}$ after injection, the rat was decapitated. A sample of the injection solution and the hemisphere ipsilateral to the injection were solubilized in duplicate in $1.5 \mathrm{ml}$ soluene-350 (Packard Instrument Co., Inc., United Technologies, Downers Grove, IL) at $50^{\circ} \mathrm{C}$ for $2 \mathrm{~h}$ before double-isotope liquid scintillation counting (12).

Counts per minute were converted to disintegrations per minute (dpm) by standard quench corrections and the percentage brain uptake index (BUI) was calculated as follows: $\mathrm{BUI}=($ test $\mathrm{dpm}$ in brain/ reference $\mathrm{dpm}$ in brain)/(test $\mathrm{dpm}$ in injectate/reference $\mathrm{dpm}$ in injectate)(100). The BUI $=E_{\mathrm{t}} / E_{\mathrm{r}}$, where $E_{\mathrm{t}}$ and $E_{\mathrm{r}}$ represent the extraction of the test compound and the reference compound $\left(\left[{ }^{14} \mathrm{C}\right]\right.$ butanol or $\left[{ }^{3} \mathrm{H}\right]$ water $)$, respectively, $15 \mathrm{~s}$ after injection. The $E_{\mathrm{t}}$ and $E_{\mathrm{r}}$ represent the extraction due to unidirectional influx into brain minus the back-diffusion of the test or reference compound during the period between bolus flow through brain $(\sim 2 \mathrm{~s}$ after injection) and decapitation (15 s after injection). With regard to the reference compounds, the extraction at $15 \mathrm{~s}$ for $\left[{ }^{14} \mathrm{C}\right]$ butanol and $\left[{ }^{3} \mathrm{H}\right]$ water under the experimental conditions is 90 and $58 \%$, respectively $(13,14)$. With regard to the test compounds, the extraction at $15 \mathrm{~s}$ is not significantly different from the initial extraction. Owing to active sequestration mechanisms in the brain for the steroid hormones and for propranolol, the half-life of ligand distribution in brain after a pulse injection is 4$6 \mathrm{~min}(14,15)$. Therefore, the ligand efflux to blood in $15 \mathrm{~s}$ is $<5 \%$. In addition, previous studies have shown that the amount of recirculating test or reference isotope is not quantitatively significant relative to the amount of radioactivity in brain for up to $4 \mathrm{~min}$ after injection (14).

Methods of $k_{3} t$ and $K_{D}{ }^{a}$ estimation. After the ligand extraction at a series of plasma protein concentrations was determined with the carotid injection technique, the following equation (derived in the Appendix) was fit to the extraction data:

$E_{\mathrm{t}}=1-\mathrm{e}^{\left(\frac{-k_{3} i}{1+A_{\mathrm{F}} / K_{\mathrm{D}}^{\mathrm{d}}}\right)}$

where $E_{\mathrm{t}}$ is the unidirectional extraction of the test compound, $k_{3}$ is the rate constant (seconds ${ }^{-1}$ ) of ligand transport through the BBB in the direction of blood to brain, $t$ is the mean brain capillary transit time (seconds), and $A_{\mathrm{F}}$ is the concentration of unoccupied plasma protein-binding sites. It is assumed that there is only one ligand binding site per plasma protein molecule. The reactions and transports that define the interactions of plasma protein, ligand, and membrane are depicted in the compartmental model shown in Fig. 1.

When no plasma protein is present, Eq. 1 is identical to the KetyRenkin-Crone (16-18) equation of capillary physiology,

$E=1-e^{-k 3 i}$,

where $k_{3} \bar{t}=P S / F$. The $P S$ product is the permeability-capillary surface area product (milliliters per minute per gram) on the blood side of the $\mathrm{BBB}$, and $F$ is the rate of cerebral blood flow (milliliters per minute per gram). The $k_{3} t=P S / F$ equality holds since by definition, $P S$ $=k_{3} \cdot V_{\mathrm{P}}$ and $F=V_{\mathrm{P}} / t$, where $V_{\mathrm{P}}$ is the capillary volume (milliliters per gram).

There are two approaches by which Eq. 1 may be fitted to the ligand extraction-plasma protein curves. In case I, two parameters, i.e., the $k_{3} t$ product and the $K_{\mathrm{D}}{ }^{2}$, are estimated by nonlinear regression analysis. In this approach, the in vivo $K_{\mathrm{D}}{ }^{2}$ is determined from the analysis of the in vivo transport data, which allow for estimation of the apparent dissociation constant in the milieu of the brain capillary. In case II, the $K_{\mathrm{D}}{ }^{2}$ is fixed at the absolute $K_{\mathrm{D}}$ value as measured in vitro by equilibrium dialysis, and only one parameter, the $k_{3} I$ product, is estimated from nonlinear regression analysis. The fundamental assumption made in the case II analyses is that the protein-ligand dissociation constant in a living capillary is identical to the absolute $K_{\mathrm{D}}$ measured in a test tube, e.g., by equilibrium dialysis. The absolute $K_{\mathrm{D}}$ values for albumin binding of corticosterone, dihydrotestosterone, testosterone, estradiol, $T_{3}$, and propranolol, and for orosomucoid binding of propranolol are listed in Table I. The absolute $K_{\mathrm{D}}$ values are determined by equilibrium dialysis at $37^{\circ} \mathrm{C}(4,5,8)$.

The fitting of Eq. 1 to extraction-plasma protein concentration curves is performed with an unweighted least squares regression analysis (subroutine PAR, BMDP programs [19]) for both the twoparameter (case I) and one-parameter (case II) studies. The $k_{3} I$ product estimated by either the case I or case II analyses is compared with the $k_{3} T$ product determined with the Kety-Renkin-Crone relationship (Eq. 2) and the ligand extraction at zero protein concentration. Alternate weighted least squares estimates were made with weights equal to the reciprocal of the squared standard errors of the observations. The results were similar, and only unweighted least squares estimate are reported. Since case II is a restriction of case I with one less

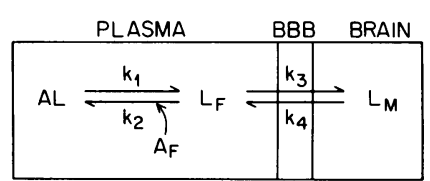

Figure 1. A compartmental model portrays the transport of protein-bound ligands into brain in vivo during passage of the bolus tracer. The brain endothelial wall, i.e., the BBB, separates the plasma and brain compartments. See Appendix for explanation of $k_{1}-k_{4}$. $A L$ protein-ligand complex. $L_{\mathrm{F}}$, free ligand in the plasma compartment. $L_{\mathrm{M}}$, free ligand in the brain compartment. Since plasma proteins do not cross the BBB, the transport of protein-bound ligands into the brain occurs via a free intermediate mechanism which involves an obligatory dissociation of protein-bound ligand into the free intermediate state before transmembrane transport. 
Table I. Comparison of Plasma Protein Dissociation Constant In Vivo in the Brain Capillary and In Vitro

\begin{tabular}{llcc}
\hline Plasma protein & Ligand & $\begin{array}{l}\text { In vitro } \\
K_{\mathrm{D}}^{*}\end{array}$ & $\begin{array}{l}\text { In vivo } \\
K_{\mathrm{D}}{ }^{*} \ddagger\end{array}$ \\
\hline \multirow{4}{*}{ Albumin } & & $\mu M$ & $\mu M$ \\
& Propranolol & $290 \pm 30$ & $220 \pm 40$ \\
& Testosterone & $53 \pm 1$ & $2,520 \pm 710$ \\
& Corticosterone & $260 \pm 10$ & $1,330 \pm 90$ \\
& Dihydrotestosterone & $53 \pm 6$ & $830 \pm 140$ \\
& Estradiol & $23 \pm 1$ & $710 \pm 100$ \\
& $T_{3}$ & $4.7 \pm 0.1$ & $46 \pm 4$ \\
Orosomucoid & Propranolol & $3.3 \pm 0.1$ & $19 \pm 4$ \\
& & & \\
\hline
\end{tabular}

* Data are mean $\pm S E$ (from references $4,5,8$ ).

‡ Estimates \pm SE from case I nonlinear regression fits.

adjustable parameter, the significance of the improvement in fit of case I over case II was assessed by an F test (20). A significant difference would indicate that the improvement in fit over case II of the case I analysis is greater than one would expect simply from making $K_{\mathrm{D}}{ }^{2}$ a second adjustable parameter, and that the case II constraint that $K_{\mathrm{D}}{ }^{\mathrm{a}}$ $=K_{\mathrm{D}}$ is inappropriate.

\section{Results}

The brain extraction of ${ }^{3} \mathrm{H}$-labeled corticosterone, dihydrotestosterone, estradiol, $T_{3}$, or propranolol is decreased when concentrations of albumin are increased (Figs. 2-6). Similarly, the brain extraction of ${ }^{3} \mathrm{H}$-propranolol is decreased when concentrations of orosomucoid are increased (Fig. 7). Figs. 2-7 also provide the predicted relationships between ligand extraction and protein concentration both for case I, where the in vivo $K_{\mathrm{D}}^{\mathrm{a}}$ is estimated from the experimental data along with the $k_{3} \bar{t}$ product, and for case II, where only the $k_{3} \bar{t}$ product is estimated and the in vivo $K_{\mathrm{D}}$ is fixed at the in vitro value. The in vivo $K_{\mathrm{D}}{ }^{2}$ estimates are shown in Table $I$ in comparison with the in vitro $K_{\mathrm{D}}$ measurements. The testosterone $K_{\mathrm{D}}{ }^{\mathrm{a}}$ was estimated from data (not shown) reported previously (4).

As shown in Figs. 2-5 and 7, the case I curves fit the experimental data much better than do the case II curves. This suggests that the case II constraint that $K_{\mathrm{D}}{ }^{\mathrm{a}}=K_{\mathrm{D}}$ is inappropriate and gives significantly poorer fits. For example, the residual sums of squares of the case I studies were 25- to 500 -fold less than the case II residual sums of squares (highly significant by appropriate $\mathrm{F}$ tests) (see Methods). Formal chisquared goodness-of-fit tests with weighted regressions (weight $=1 /[\text { standard error }]^{2}$ ) revealed that the weighted residual sums of squares for the case I fits were consistent with the known experimental error. In addition, the $k_{3} \bar{t}$ products estimated from the case I curves agree much better with the $k_{3} \bar{t}$ products computed with Eq. 2 and the extraction of ligand at zero protein concentration. For example, the extraction of dihydrotestosterone, estradiol, corticosterone, $\mathrm{T}_{3}$, and propranolol at zero protein concentration is $93 \pm 5,85 \pm 3,39 \pm 2,13 \pm 1$, and $68 \pm 2 \%$, respectively (Figs. 2-6). Substitution of these extraction values into Eq. 2 indicates that the $k_{3} \bar{t}$ product for dihydrotestosterone, estradiol, corticosterone, $\mathrm{T}_{3}$, and propranolol is 2.7 , $1.9,0.48,0.14$, and 1.1 , respectively. These estimates are nearly identical to the case I estimates of the $k_{3} \bar{t}$ product (Figs. 2-7).

If it is assumed that Eq. 2 provides accurate estimates of the $k_{3} \bar{t}$ product and that the $K_{\mathrm{D}}{ }^{\mathrm{a}}$ in vivo does not deviate from the in vitro $K_{\mathrm{D}}$ value, then the ligand extraction at a given albumin concentration may be computed with Eq. 1. The dotted line on the case I side of Figs. 2-5 and 7 shows the

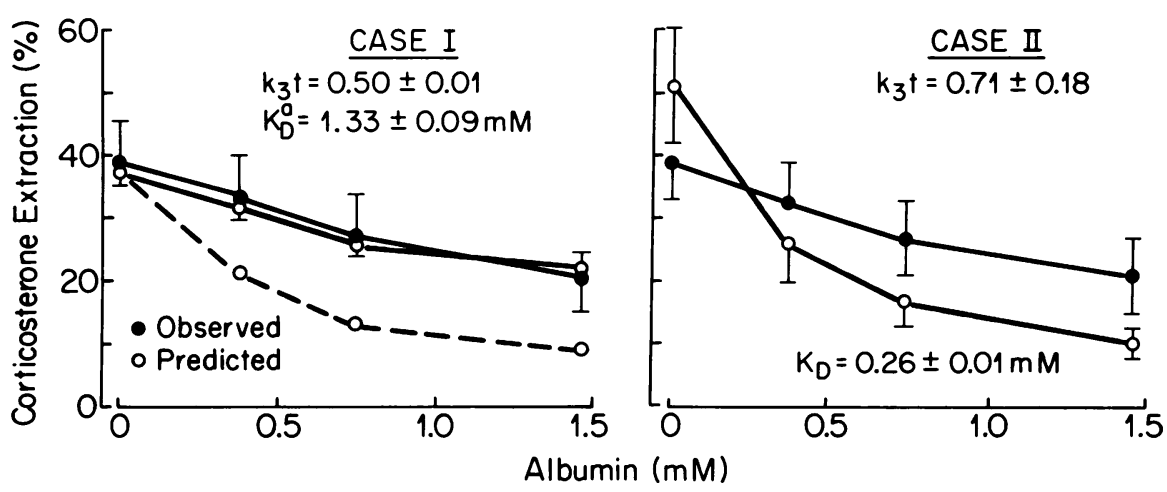

Figure 2. The unidirectional extraction of $\left[{ }^{3} \mathrm{H}\right]$ corticosterone by rat brain is plotted versus the arterial albumin concentration. The experimentally observed values reported in reference 4 are given by the closed circles (mean \pm SE, $n=3-6$ animals per point). The extraction values \pm SD predicted on the basis of fitting the experimental data to Eq. 1 (see Methods) is given in open circles. Case I involves the estimation of two parameters from the nonlinear regression analysis of Eq. 1. These parameters are the $k_{3} I$ product and the in vivo $K_{\mathrm{D}}^{2}$, where $K_{\mathrm{D}}=k_{1} /$ $k_{2}$. Case II involves the estimation of only one parameter from the nonlinear regression analysis of Eq. 1, i.e., the $k_{3} I$ product, where the $K_{\mathrm{D}}$ is fixed at the in vitro value, $0.26 \pm 0.01 \mathrm{mM}$. The latter value is the $K_{\mathrm{D}}$ estimated by equilibrium dialysis (4). The case I fit was significantly improved over the case II fit $(P=0.0002$ by the F-test; see Methods). The dotted line on the case I side of the figure represents the extraction values predicted by substituting into Eq. 1 the respective albumin concentration, the $k_{3} \bar{t}$ estimated by Eq. 2 , and the $K_{\mathrm{D}}$ determined by equilibrium dialysis. 


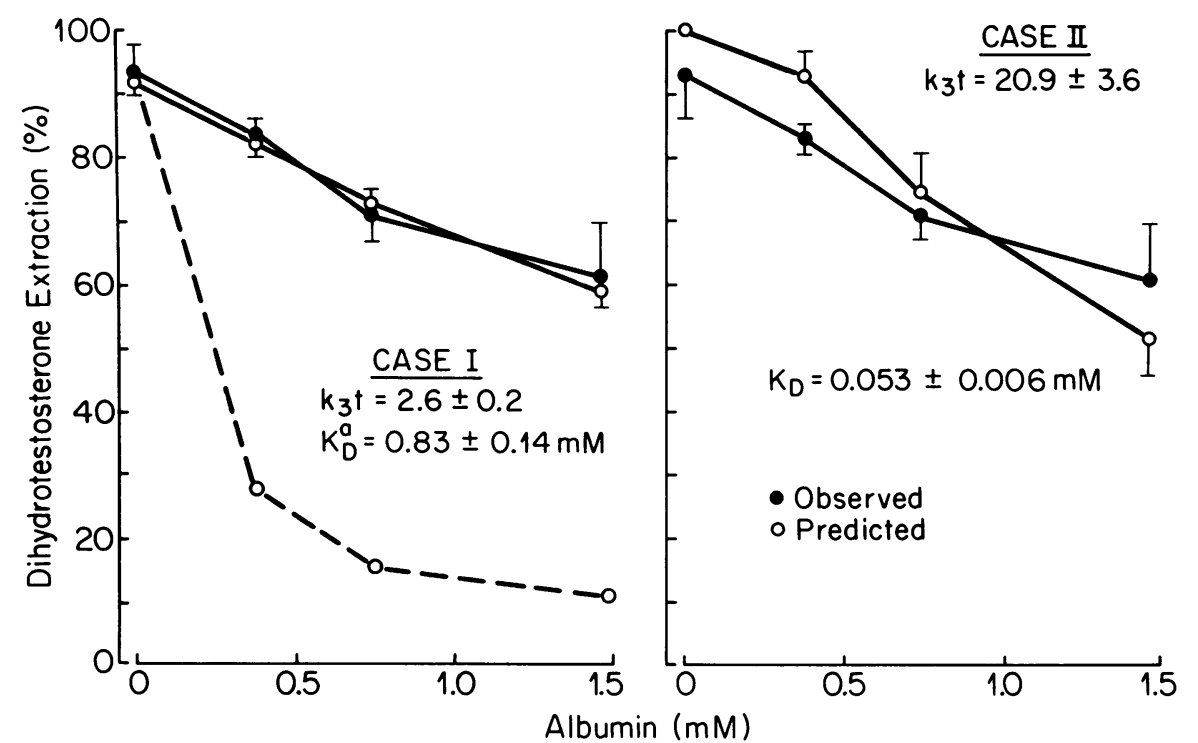

Figure 3. The unidirectional extraction of $\left[{ }^{3} \mathrm{H}\right]$ dihydrotestosterone by brain is plotted versus the albumin concentration. See legend to Fig. 2 for experimental details and the differences between the case I and case II regression analyses. The case I fit was significantly improved over the case II fit ( $P=0.02$ by the F-test; see Methods). expected extraction for each ligand given the respective values for the in vitro $K_{\mathrm{D}}$ and the values for $k_{3} \bar{t}$ calculated with Eq. 2 . These curves reveal that if no change in $K_{\mathrm{D}}{ }^{\mathrm{a}}$ occurs in vivo, then the inhibition of brain extraction of ligand exerted by albumin should be much greater than what is observed experimentally (Figs. 2-5 and 7).

The effects of albumin on brain extraction of propranolol are described in essentially an identical way by either the case I or case II analyses (Fig. 6). Both approaches give identical estimates of $k_{3} \bar{t}$, and the in vivo $K_{\mathrm{D}}{ }^{\mathrm{a}}$ estimated by the case I analysis is not significantly different from the in vitro $K_{\mathrm{D}}$ (case II, Fig. 6). The data in Fig. 6 provide an important control for the plots in Figs. 2-5 and 7 and indicate that the analysis used in these studies can in one case achieve confirming estimates of the $K_{\mathrm{D}}$ as measured either in vivo in brain capillaries or in vitro by equilibrium dialysis.

\section{Discussion}

The present studies constitute a quantitative analysis of previously reported data within the context of a mathematical model (Fig. 1) which leads to the derivation of Eq. 1. This equation represents a modified form of the Kety-Renkin-Crone (16-18) equation of capillary physiology and is useful for studying capillary transport of plasma protein-bound ligands. The fitting of experimental transport data to Eq. 1 under the

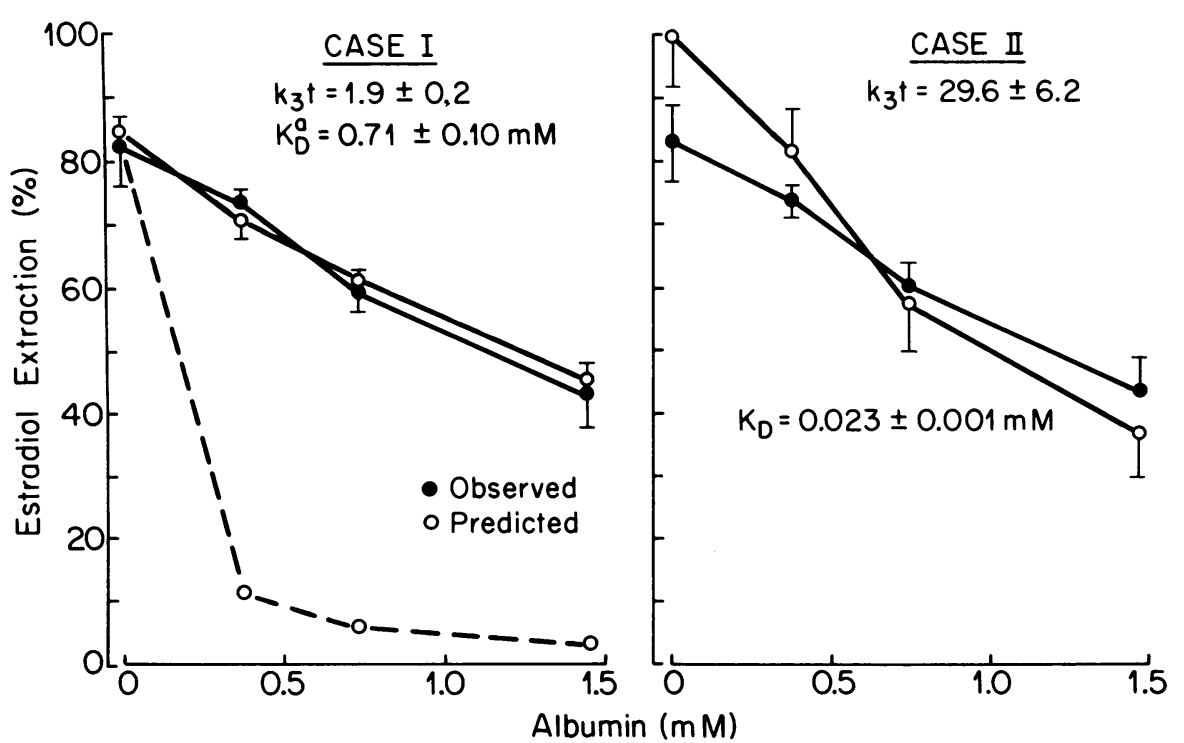

Figure 4. The unidirectional extraction of $\left[{ }^{3} \mathrm{H}\right]$ estradiol by brain is plotted versus the arterial albumin concentration. See legend to Fig. 2 for experimental details for the case I and case II regression analyses. The experimentally observed values are from reference 4 . The case I fit was significantly improved over the case II fit $(P=0.02$ by the F-test; see Methods). 


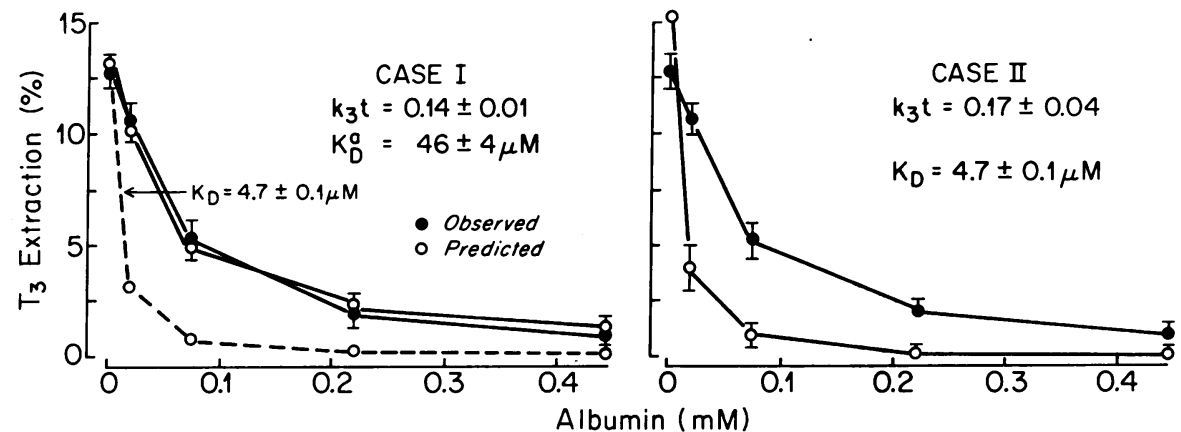

Figure 5. The unidirectional extraction of $\left.{ }^{125} \mathrm{I}\right] \mathrm{T}_{3}$ by rat brain is plotted versus the arterial albumin concentration. See legend to Fig. 2 for experimental details of the case I and case II regression analyses. The experimentally observed values are from reference 5 . The case I fit was significantly improved over the case II fit $(P<0.0001$ by the F-test; see Methods). restriction of the free hormone or drug hypothesis (i.e., $K_{\mathrm{D}}{ }^{\mathrm{a}}$ $=K_{\mathrm{D}}$, case II studies) shows that poor data fits are obtained and overestimations of $k_{3} \bar{t}$ products are generated. Rather, we find that, in most cases, good data fits to Eq. 1 and appropriate estimates of $k_{3} \bar{t}$ are obtained if the in vivo dissociation constant, $K_{\mathrm{D}}$ a , is many times greater than the in vitro $K_{\mathrm{D}}$ (case I studies, Table I). The high values for $K_{\mathrm{D}}{ }^{2}$ in vivo suggest that ligand dissociation from plasma protein is greatly enhanced in the microcirculation. The increased dissociation of ligand in the microcirculation allows for the transport of protein-bound ligand into the tissue, without allowing the plasma protein to leave the capillary space (9).

The estimation of plasma protein-ligand binding constants in vivo with a bolus injection technique is based on the validity of at least six assumptions. First, it is assumed that blood-to-brain transport processes measured during a single capillary transit time, $\sim 1 \mathrm{~s}$, may be modeled by a compartmental approach (Fig. 1). Second, it is assumed that the ligandprotein binding reaction remains at near-equilibrium during the course of bolus passage through the brain. Third, no dilution artifacts are caused by a mixing of the injection bolus with the circulating rat plasma. Fourth, ligand-protein interactions are linear or first order. Fifth, membrane transport of free ligand is first order at the concentration of labeled ligand used in these studies. Sixth, the brain microcirculation is endowed with a specific mechanism that leads to enhanced dissociation of the ligand from the protein, such that proteinbound hormone is available for transport through the membrane, while the plasma protein remains in the capillary plasma space.

The use of the compartmental model in Fig. 1 assumes (a) that events occurring over a wide area of the brain capillary bed may be averaged so that the plasma compartment behaves like a well-mixed pool, and $(b)$ that the bolus of tracer behaves as though it were in fixed contact with the BBB for a period of time, $\bar{t}^{2}$ Since the radius of cells is greater than the capillary

2. An alternate partly compartmental-partly flow system model, in which the bolus is treated as clearing the microcirculation through a single exponential washout, gave similar findings when case I and case II fits were compared. radius, plug or bolus flow, but not laminar flow, is present within the capillary (21). Therefore, it is unlikely that significant radial diffusion effects occur. We have implicitly assumed that longitudinal (axial) diffusion effects are negligible. The need for a more complex model $(22,23)$ that may account for longitudinal gradients in the capillary bed or distributions of capillary lengths is untested with regard to the present studies.

A bolus injection technique may be used quantitatively to assess in vivo plasma protein binding if the binding reaction between ligand and plasma protein is in near-equilibrium during the course of the bolus transit through the capillary (21). This assumption requires that binding reactions are fast relative to the transport reaction, i.e., $k_{2} A_{\mathrm{F}}$ and/or $k_{1} \gg k_{3}$ and $k_{4}$ (see Appendix). Because $k_{3} \bar{t}$ products range from 0.14 to 2.6 and $t$ is approximately equal to $1 \mathrm{~s}, k_{3}$ ranges from 0.14 to 2.6. Since $A_{\mathrm{F}}=10-1,500 \mu \mathrm{M}$ (Figs. 2-7), it is assumed that $k_{2} \gg 10^{2}-10^{4} \mathrm{M}^{-1} \mathrm{~s}^{-1}$. The minimum value for hormoneplasma protein association reactions is $10^{6}-10^{8} \mathrm{M}^{-1} \mathrm{~s}^{-1}$ (24). Therefore, the assumption of near-equilibrium for the binding reaction appears justified (4).

The assumption that there is insignificant mixing of the bolus with the circulating rat plasma may be attributed to the fact that the rate of carotid injection $(>0.3 \mathrm{ml} / \mathrm{s}$ or $>18 \mathrm{ml} /$ $\mathrm{min}$ ) exceeds the rate of common carotid blood flow $(5 \mathrm{ml}$ / $\mathrm{min}$ ) in the barbiturate-anesthetized rat (25). The bolus nature of the passage of the injection solution through brain is supported by the observation that $96 \%$ of the injection solution has already cleared the brain by $2 \mathrm{~s}$ after injection (12). The maintenance of a bolus for at least one circulation is also shown in the early studies of Paton (see Fig. 1 of reference 26). In addition, from our previous studies of BBB transport of glucose and amino acids, there is direct experimental support for the assumption of insignificant bolus mixing. For example, the inclusion of $90 \%$ serum in the carotid artery injection solution results in $85 \%$ inhibition of the extraction of arginine, an amino acid that is transported through the BBB by a specific transport system that is heavily saturated by normal plasma amino acid concentrations $(27,28)$. When glucose or amino acid transport through the BBB is measured after carotid injection of the substances in Ringer's solution, the observed permeability constant is the value expected if no 


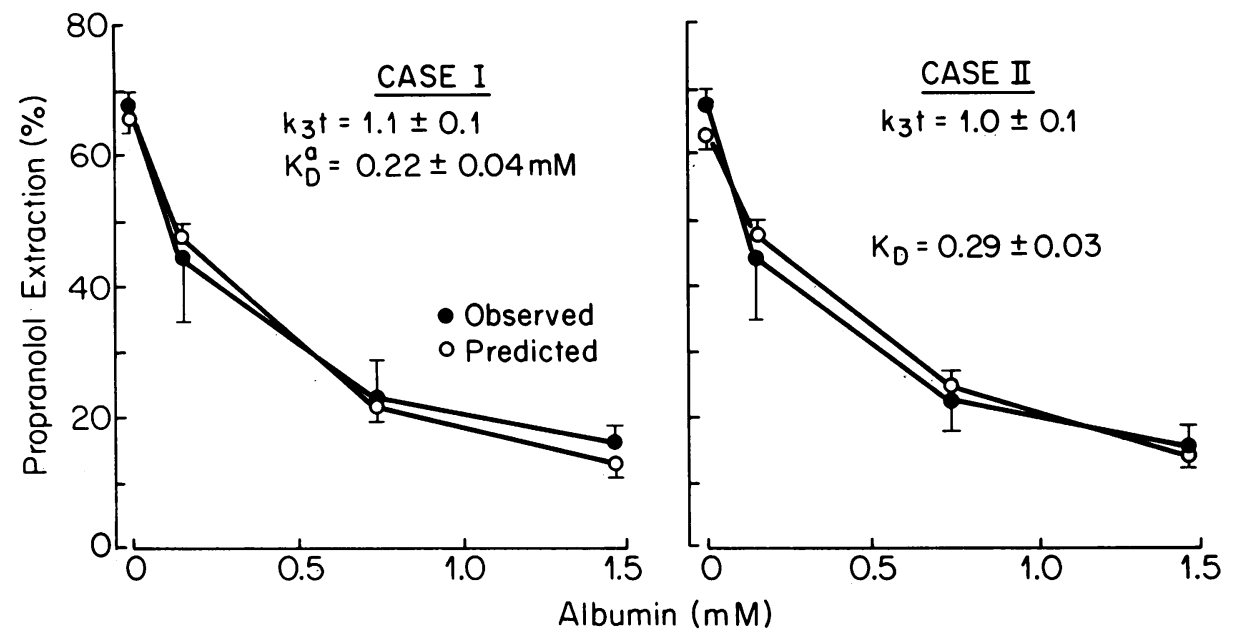

Figure 6. The unidirectional extraction of $\left[{ }^{3} \mathrm{H}\right]$ propranolol by brain is plotted versus the arterial albumin concentration. See legend to Fig. 2 for experimental details for the case I and case II regression analyses. The experimentally observed values are from reference 8 . The dotted line plot (e.g., Figs. 2-5) is not shown in this figure as this plot, which represents the case where $k_{3} \bar{t}$ $=1.1$ and $K_{\mathrm{D}}^{\mathrm{a}}=0.29 \mathrm{mM}$, essentially overlaps the predicted and observed curves in the figure. The case I fit was not significantly improved over the case II fit ( $P=0.6$ by the F-test; see Methods). competing endogenous substrates are present in the brain microcirculation (28). Moreover, a mixing artifact, if present, should be comparable for all ligands and plasma proteins. The data in Fig. 6 indicate that our experimental approach can, in some cases, give results consistent with the free hormone or drug hypothesis.

The assumptions about linearity of the binding and transport reactions appear reasonable since $(a)$ the concentration of tracer hormone or drug used in these studies is $<0.1 \mu \mathrm{M}$, which is small compared with the minimum $K_{\mathrm{D}}$ of the binding proteins used (Table I), and $(b)$ membrane transport is nonsaturable for all the ligands studied when ligand concentration is $<0.1 \mu \mathrm{M}(4,5,6,15)$.

Given the validity of the above assumptions, we conclude that ligand binding to plasma proteins is, in most cases, altered in the microcirculation so that the system operationally behaves as if the dissociation constant of the binding reaction is greatly increased relative to the in vitro situation. One possible mechanism by which this might happen is the release from the endothelial surface of noncompetitive plasma protein-binding inhibitors. The putative inhibitor might be free fatty acids, since Chopra et al. (29) have recently suggested that these molecules inhibit plasma protein binding of thyroid hormones. The interaction between the plasma protein and the putative inhibitor may cause a conformational change in the plasma protein that results in enhanced dissociation of the ligand from the plasma protein. Moreover, the putative conformational change in albumin probably involves only some of the albumin binding sites, e.g., the steroid or thyroid hormone binding sites, and not other parts of the albumin molecule. The propranolol binding site appears to be unaffected by albumin interactions with the microcirculation, since the $K_{\mathrm{D}}{ }^{\mathrm{a}}=K_{\mathrm{D}}$ for

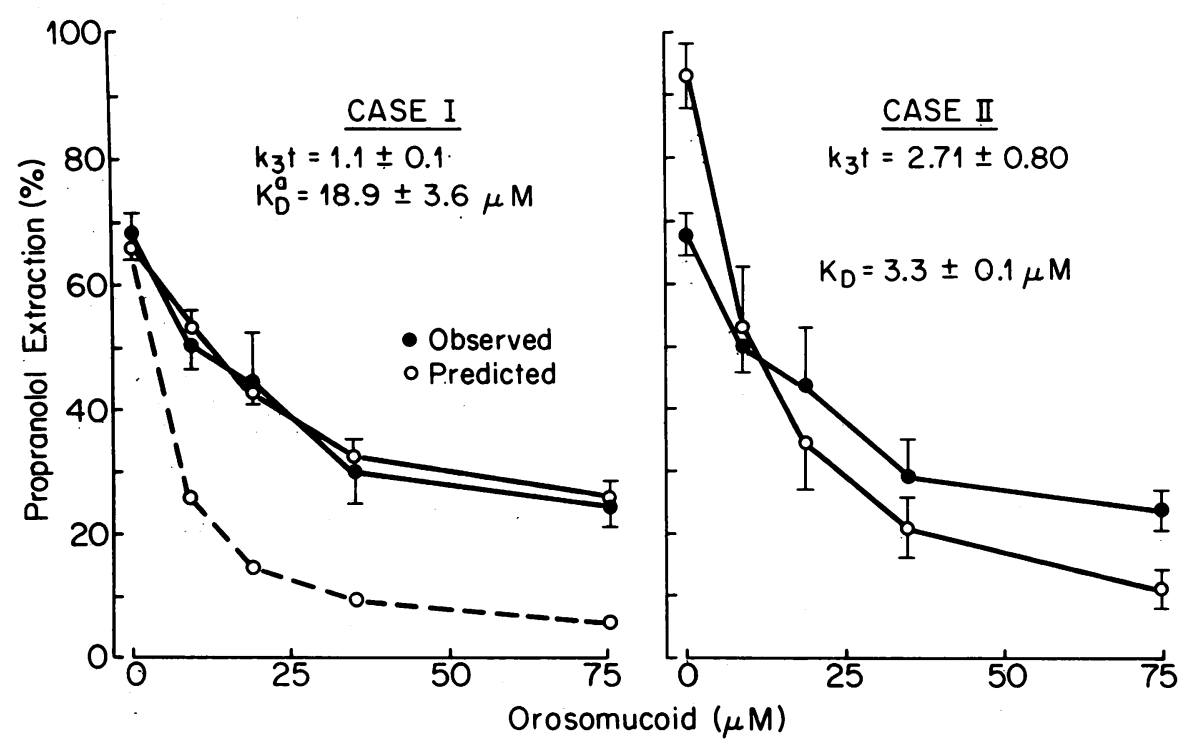

Figure 7. The unidirectional extraction of $\left[{ }^{3} \mathrm{H}\right]$ propranolol by rat brain is plotted versus the arterial orosomucoid concentration. See legend to Fig. 2 for experimental details of the case I and case II regression analyses. The experimentally observed values are from reference 8 . The case I fit was significantly improved over the case II fit $(P=0.002$ by the F-test; see Methods). 
albumin binding of propranolol (Fig. 6). Similarly, albuminbound phenobarbital is not available for transport through the adult rat $\mathrm{BBB}(30)$

Another possible mechanism by which albumin-bound ligands may be transported into tissues is a receptor-mediated process. Weisiger et al. (31) and Forker and Luxon (32) propose that albumin binds to a hepatocyte surface receptor that has a relatively high affinity for albumin, e.g., a dissociation constant of 25-50 $\mu \mathrm{M}$ albumin. The evidence for the receptor hypothesis is, in part, the observed decrease in the rate of transport of protein-bound ligand caused by increasing concentrations of albumin $(31,32)$. We observe this same saturation phenomenon (Figs. 2-7). However, we find that the extent to which the transport of albumin-bound ligands is saturated by the plasma protein is quite variable from ligand to ligand (Table I). If albumin-bound ligands are transported through the BBB via a single endothelial albumin receptor, it would be expected that the saturation of the process by albumin is comparable for all albumin-bound ligands. With regard to BBB transport of protein-bound ligands, it is not possible at present to discriminate between an inhibitor model and a receptor model. Both of these possible mechanisms are the subject of our present laboratory investigation.

\section{Appendix}

Mathematical model. These studies employ a compartmental model depicting the transport of a protein-bound ligand through the BBB and into the brain (Fig. 1). If a relatively fixed concentration of free protein is assumed, the differential equations of the model are

$V_{\mathrm{P}} \frac{\mathrm{d} A L}{\mathrm{~d} t}=k_{2} A_{\mathrm{F}} L_{\mathrm{F}} V_{\mathrm{P}}-k_{1}(A L) V_{\mathrm{P}}$,

$V_{\mathrm{P}} \frac{\mathrm{d} L_{\mathrm{F}}}{\mathrm{d} t}=k_{1}(A L) V_{\mathrm{P}}+k_{4} L_{\mathrm{M}} V_{\mathrm{T}}-\left(k_{2} A_{\mathrm{F}}+k_{3}\right) L_{\mathrm{F}} V_{\mathrm{P}}$, and

$V_{\mathrm{T}} \frac{\mathrm{d} L_{\mathrm{M}}}{\mathrm{d} t}=k_{3} L_{\mathrm{F}} V_{\mathrm{P}}-k_{4} L_{\mathrm{M}} V_{\mathrm{T}}$,

where $A L$ is the concentration of the albumin-ligand complex in plasma (M), $L_{\mathrm{F}}$ is the concentration of the free ligand in plasma (M), $L_{\mathrm{M}}$ is the concentration of ligand in brain $(\mathrm{M}), A_{\mathrm{F}}$ is the concentration of free or unoccupied protein binding sites in plasma (M), $k_{1}$ is the rate constant of ligand dissociation (seconds ${ }^{-1}$ ), $k_{2}$ is the rate constant of ligand association (molar ${ }^{-1}$ seconds $\left.{ }^{-1}\right), k_{3}$ is the rate constant of plasma to brain transport through the $\mathrm{BBB}$ (seconds ${ }^{-1}$ ), $k_{4}$ is the rate constant of brain to plasma transport through the BBB (seconds ${ }^{-1}$ ), $V_{\mathrm{P}}$ is the brain capillary blood volume (liters per kilogram), and $V_{\mathrm{T}}$ is the brain tissue apparent volume of distribution (liters per kilogram). The initial conditions are $A L(0)=A L^{0} ; L_{\mathrm{F}}(0)=L_{\mathrm{F}}{ }^{\circ} ; L_{\mathrm{M}}(0)=0$; and $L_{\mathrm{T}}$ $=A L^{\circ}+L_{\mathrm{F}}^{\circ}$, where $L_{\mathrm{T}}=$ the initial total ligand concentration in plasma (M).

The equations that define $L_{\mathrm{M}}(t), L_{\mathrm{F}}(t)$, and $A L(t)$ may be simplified to approximate solutions if specific restrictions on the rate constants are applied. If it is assumed that $k_{2} A_{\mathrm{F}}$ and/or $k_{1} \gg k_{3}$ and $k_{4}$, then the system of equations becomes relatively stiff such that the ligand-protein dynamics is much faster than the transport kinetics. Therefore, in the transport time scale, Eq. 3 is in approximate equilibrium, and $A L(t)$
$\simeq\left[A_{\mathrm{F}} L_{\mathrm{F}}(t)\right] / K_{\mathrm{D}} \mathrm{a}$, where the apparent in vivo dissociation constant, $K_{\mathrm{D}}{ }^{\mathrm{a}}$, is equal to $k_{1} / k_{2}$. The ligand mass conservation equation is defined by $L_{\mathrm{T}}=L_{\mathrm{F}}(t)+L_{\mathrm{M}}(t) \frac{V_{\mathrm{T}}}{V_{\mathrm{P}}}+A L(t)$. Substitution of the approximate solution for $A L(t)$ into the ligand conservation equation results in

$L_{\mathrm{F}}(t)=\frac{L_{\mathrm{T}}-L_{\mathrm{M}}(t)}{1+A_{\mathrm{F}} / K_{\mathrm{D}}^{\mathrm{a}}}\left(V_{\mathrm{T}} / V_{\mathrm{P}}\right)$.

Substitution of Eq. 6 into Eq. 5 and rearrangement result in

$$
\left(\frac{V_{\mathrm{T}}}{V_{\mathrm{P}}}\right) \frac{\mathrm{d} L_{\mathrm{M}} / \mathrm{d} t}{L_{\mathrm{T}}}=\left(\frac{k_{3}}{1+A_{\mathrm{F}} / K_{\mathrm{D}}{ }^{2}}\right)-\left(\frac{k_{3}}{1+A_{\mathrm{F}} / K_{\mathrm{D}}{ }^{2}}+k_{4}\right)\left[\frac{L_{\mathrm{M}}(t)}{L_{\mathrm{T}}}\right] \frac{V_{\mathrm{T}}}{V_{\mathrm{P}}} \text {. }
$$

Letting the extraction of the ligand by the tissue be defined by

$E(t)=\frac{\left[L_{\mathrm{M}}(t)\right]}{L_{\mathrm{T}} V_{\mathrm{P}}} V_{\mathrm{T}}$

and integrating Eq. 7, with $L_{M}(0)=0$, gives

$E=\left[\frac{1}{1+k_{4}\left(1+A_{\mathrm{F}} / K_{\mathrm{D}}^{\mathrm{a}}\right) / k_{3}}\right]\left\{1-\mathrm{e}^{-\left(k_{4}+k_{3} /\left(1+A_{\mathrm{F}} / K_{\mathrm{D}}\right)\right) k^{2}}\right\}$.

Eq. 9 may be further simplified if it is assumed that

$k_{4}\left(1+A_{\mathrm{F}} / K_{\mathrm{D}}^{2}\right) \ll k_{3}$ (footnote 3 ).

Therefore, if the time variable is set equal to the mean transit time, $\bar{t}$, Eq. 9 reduces to Eq. 1 of the text

$E=1-\mathrm{e}^{-k \mathbf{3} /\left(1+A F / K D^{\infty}\right)}$.

\section{Acknowledgments}

Larry Mietus and Gary Fierer provided excellent technical assistance. Janice Brothers provided outstanding secretarial assistance. Dr. Joseph J. DiStefano III provided valuable advice and suggestions. The authors are grateful to one of the referees for a critical analysis of the manuscript.

These studies are supported by National Institutes of Health grant AM-25744. E. M. Landaw is supported by National Science Foundation grant ECS-80-15965 and National Institutes of Health grant CA-16042.

\section{References}

1. Robbins, J., J. E. Rall, and P. Gorden. 1974. The thyroid and iodine metabolism. In Duncan's Diseases of Metabolism. P. K. Bondy

3. If ligand transport is symmetrical, i.e., PS (blood side) = PS (brain side), then the equality $k_{4}=k_{3}\left(V_{\mathrm{P}} / V_{\mathrm{T}}\right)$ holds (PS, permeability-capillary surface area product; $V_{\mathrm{T}}$, brain tissue apparent volume of distribution). Since the blood volume in brain, $V_{\mathrm{P}}$, is $0.02 \mathrm{ml} \mathrm{g}^{-1}(33)$, and the brain water space is $0.7 \mathrm{ml} \mathrm{g}^{-1}(34)$, then a maximum $V_{\mathrm{P}} / V_{\mathrm{T}}$ of $k_{4} / k_{3}$ ratio is 0.03 . However, the $k_{4} / k_{3}$ ratio may be considerably less than 0.03 for substances that are actively sequestered by intracellular binding systems in brain. Moreover, the gonadal steroid hormones and lipophilic amine drugs such as propranolol have been previously shown to be bound by rat brain $(14,15)$. Therefore, the assumption given in Eq. 10 easily holds for all the case I studies and is reasonably valid for the case II analyses. 
and L. E. Rosenberg, editors. W. B. Saunders Co., Philadelphia. 10091104.

2. Ekins, R. P. 1979. Methods for the measurement of free thyroid hormones. In Free Thyroid Hormones. R. Ekins, G. Faglia, F. Pennisi, and A. Pinchera, editors. Excerpta Medica, Amsterdam. 72-92.

3. Koch-Weser, J., and E. M. Sellers. 1976. Binding of drugs to serum albumin. N. Engl. J. Med. 294:311-316.

4. Pardridge, W. M., and L. J. Mietus. 1979. Transport of steroid hormones through the rat blood-brain barrier. Primary role of albuminbound hormone. J. Clin. Invest. 64:145-154.

5. Pardridge, W. M. 1979. Carrier-mediated transport of thyroid hormones through the rat blood-brain barrier: primary role of albuminbound hormone. Endocrinology. 105:605-612.

6. Pardridge, W. M., and L. J. Mietus. 1980. Transport of thyroid and steroid hormones through the blood-brain barrier of the newborn rabbit: primary role of protein-bound hormone. Endocrinology. 107:1705-1710.

7. Pardridge, W. M., and L. J. Mietus. 1980. Palmitate and cholesterol transport through the blood-brain barrier. J. Neurochem. 34:463-466.

8. Pardridge, W. M., R. Sakiyama, and G. Fierer. 1983. Transport of propranolol and lidocaine through the rat blood-brain barrier. Primary role of globulin-bound drug. J. Clin. Invest. 71:900-908.

9. Pardridge, W. M. 1981. Transport of protein-bound hormones into tissues in vivo. Endocr. Rev. 2:103-123.

10. Robbins, J., and M. L. Johnson. 1982. Possible significance of multiple transport proteins for the thyroid hormones. In Free Hormones in Blood. A. Albertini and R. P. Ekins, editors. Elsevier/North Holland Biomedical Press BV, Amsterdam. 53-64.

11. Ekins, R., P. Edwards, and B. Newman. 1982. The role of binding proteins in hormone delivery. In Free Hormones in Blood. A. Albertini and R. P. Ekins, editors. Elsevier/North Holland Biomedical Press BV, Amsterdam. 3-43.

12. Oldendorf, W. H., and L. D. Braun. 1976. $\left({ }^{3} \mathrm{H}\right)$-Tryptamine and ${ }^{3} \mathrm{H}$-water as diffusible internal standards for measuring brain extraction of radio-labeled substances following carotid injection. Brain Res. 113:219-224.

13. Pardridge, W. M., P. D. Crane, L. J. Mietus, and W. H. Oldendorf. 1982. Kinetics of regional blood-brain barrier transport and brain phosphorylation of glucose and 2-deoxyglucose in the barbiturate-anesthetized rat. J. Neurochem. 38:1413-1418.

14. Pardridge, W. M., T. L. Moeller, L. J. Mietus, and W. H. Oldendorf. 1980. Blood-brain barrier transport and brain sequestration of steroid hormones. Am. J. Physiol. 239:E96-E102.

15. Pardridge, W. M., R. Sakiyama, and G. Fierer. 1984. Bloodbrain barrier transport and brain sequestration of propranolol and lidocaine. Am. J. Physiol. 247:R582-R588.

16. Kety, S. S. 1951. The theory and applications of the exchange of inert gas at the lungs and tissues. Pharmacol. Rev. 3:1-41.
17. Renkin, E. M. 1959. Transport of potassium- 42 from blood to tissue in isolated mammalian skeletal muscles. Am. J. Physiol. 197:12051210.

18. Crone, C. 1973. Permeability of capillaries in various organs as determined by use of the "indicator diffusion" method. Acta Physiol. Scand. 58:292-305.

19. Dixon, W. J. 1981. BMDP Statistical Software. University of California Press, Berkeley.

20. Beck, J. V., and K. J. Arnold. 1977. Parameter Estimation in Engineering and Science. John Wiley and Sons, New York. 386.

21. Lassen, N. A., and W. Perl. 1979. Tracer Kinetic Methods in Medical Physiology. Raven Press, New York.

22. Goresky, C. A. 1982. The processes of cellular uptake and exchange in the liver. Fed. Proc. 40:3033-3039.

23. Bassingthwaighte, J. B. 1974. A concurrent flow model for extraction during transcapillary passage. Circ. Res. 35:483-503.

24. Westphal, U. 1978. Steroid binding globulins: recent results. In Receptors and Hormone Action. Vol. 2. B. W. O'Malley, editor. Academic Press, New York. 443-472.

25. Ross, G., F. N. White, A. W. Brown, and A. Kolin. Regional blood flow in the rat. J. Appl. Physiol. 21:1273-1275.

26. Paton, W. D. M. 1960. The principles of drug action. Proc. Royal Soc. Med. 53:29-44.

27. Oldendorf, W. H. 1971. Brain uptake of radiolabeled amino acids, amines, and hexoses after arterial injection. Am. J. Physiol. 221:1629-1.

28. Pardridge, W. M. 1983. Brain metabolism: a perspective from the blood-brain barrier. Physiol. Rev. 63:1481-1535.

29. Chopra, I. J., T. S. Huang, R. E. Hurd, A. Beredo, and D. H. Solomon. 1984. A competitive ligand binding assay for measurement of thyroid hormone binding inhibitor in serum and tissues. J. Clin. Endocrinol. Metab. 58: 619-628.

30. Cornford, E. M., W. M. Pardridge, L. D. Braun, and W. H. Oldendorf. 1983. Increased blood-brain barrier transport of proteinbound anticonvulsant drugs in the newborn. J. Cereb. Blood Flow Metab. 3:280-286.

31. Weisiger, R., J. Gollan, and R. Ockner. 1981. Receptor for albumin on the liver cell surface may mediate uptake of fatty acids and other albumin-bound substances. Science (Wash. DC). 211:10481051.

32. Forker, E. L., and B. A. Luxon. 1983. Albumin-mediated transport of rose bengal by perfused rat liver. Kinetics of the reaction at the cell surface. J. Clin. Invest. 72:1764-1771.

33. Blasberg, R. G., J. D. Fenstermacher, and C. S. Patlak. 1983. Transport of $\alpha$-amino isobutyric acid across brain capillary and cellular membranes. J. Cereb. Blood Flow Metab. 3:8-32.

34. Crane, P. D., L. D. Braun, E. M. Cornford, J. E. Cremer, J. M. Glass, and W. H. Oldendorf. 1978. Dose dependent reduction of glucose utilization by pentobarbital in rat brain. Stroke. 9:12-18. 